\title{
A novel histone deacetylase inhibitor, Scriptaid, induces growth inhibition, cell cycle arrest and apoptosis in human endometrial cancer and ovarian cancer cells
}

\author{
NORIYUKI TAKAI, TAMI UEDA, MASAKAZU NISHIDA, KAEI NASU and HISASHI NARAHARA \\ Department of Obstetrics and Gynecology, Oita University Faculty of Medicine, Oita, Japan
}

Received October 3, 2005; Accepted November 11, 2005

\begin{abstract}
Histone deacetylase inhibitors (HDACIs) can inhibit cell proliferation, induce cell cycle arrest, and stimulate the apoptosis of cancer cells. We investigated the effects of a novel HDACI, Scriptaid, on the Ishikawa endometrial cancer cell line, SK-OV-3 ovarian cancer cell line, and normal human endometrial epithelial cells. Endometrial and ovarian cancer cells were treated with various concentrations of Scriptaid, and its effect on cell growth, cell cycle, apoptosis, and related measurements was investigated. 3-(4,5-dimethylthiazol-2yl)-2,5-diphenyltetrazolium bromide assays showed that all endometrial and ovarian cancer cell lines were sensitive to the growth inhibitory effect of Scriptaid, although normal endometrial epithelial cells were viable after treatment with the same doses of Scriptaid that induced the growth inhibition of endometrial and ovarian cancer cells. Cell cycle analysis indicated that their exposure to Scriptaid decreased the proportion of cells in the $\mathrm{S}$ phase and increased the proportion in the G0/G1 and/or G2/M phases of the cell cycle. Induction of apoptosis was confirmed by annexin $\mathrm{V}$ staining of externalized phosphatidylserine and loss of the transmembrane potential of mitochondria. This induction occurred in concert with the altered expression of genes related to cell growth, malignant phenotype, and apoptosis. Furthermore, Scriptaid treatment of these cell lines increased acetylation of $\mathrm{H} 3$ and $\mathrm{H} 4$ histone tails. These results raise the possibility that Scriptaid may prove particularly effective in the treatment of endometrial and ovarian cancers.
\end{abstract}

\section{Introduction}

One of the most important mechanisms in chromatin remodeling is the post-translational modification of the $\mathrm{N}$ terminal tails of histones by acetylation, which contributes to

Correspondence to: Dr Noriyuki Takai, Department of Obstetrics and Gynecology, Oita University Faculty of Medicine, 1-1 Idaigaoka, Hasama-machi, Oita 879-5593, Japan

E-mail: takai@med.oita-u.ac.jp

Key words: cell cycle, apoptosis, acetylation, endometrial cancer, ovarian cancer a 'histone code' determining the activity of target genes (1). Transcriptionally silent chromatin is composed of nucleosomes in which the histones have low levels of acetylation on the lysine residues of their amino-terminal tails. Acetylation of histone proteins neutralizes the positive charge on lysine residues and disrupts the nucleosome structure, allowing an unfolding of the associated DNA with subsequent access by transcription factors, resulting in changes in gene expression.

Acetylation of core nucleosomal histones is regulated by the opposing activities of histone acetyltransferases (HATs) and histone deacetylases (HDACs). HDACs catalyze the removal of acetyl groups on the amino-terminal lysine residues of core nucleosomal histones, and this activity is generally associated with transcriptional repression. Aberrant recruitment of HDAC activity has been associated with the development of certain human cancers (2). Transcription factors, such as Mad-1, BCL-6, and ETO, have also been shown to assemble HDAC-dependent transcriptional repressor complexes (3-5).

HDAC inhibitors (HDACIs), such as trichostatin A (TSA) and sodium butyrate $(\mathrm{NaB})$, can inhibit cancer cell growth in vitro (6) and in vivo (7), revert oncogene-transformed cell morphology (8), induce apoptosis (9), and enhance cell differentiation (10). Several classes of HDACIs have been identified, including: a) short-chain fatty acids [e.g. butyrates and valproic acid (VPA)]; b) organic hydroxamic acids [e.g. TSA and suberoyl anilide bishydroxamine (SAHA)]; c) cyclic tetrapeptides (e.g. trapoxin); and d) benzamides (e.g. MS275) (11). The structure of SAHA is related to that of TSA, a natural product isolated from Streptomyces hygroscopicus that was initially used as an antifungal antibiotic (11). Phenylbutyrate has been used as a single agent in the treatment of ß-thalassemia, toxoplasmosis, and malaria.

Some HDACIs (e.g. TSA and trapoxin) are of limited therapeutic use due to poor bioavailability in vivo as well as toxic side effects at high doses. $\mathrm{NaB}$ and phenylbutyrate are degraded rapidly after i.v. administration and therefore require high doses exceeding $400 \mathrm{mg} / \mathrm{kg} /$ day (12). Furthermore, these compounds are not specific for HDACs, as they also inhibit the phosphorylation and methylation of proteins as well as DNA methylation (13).

Synthetic analogs isolated from screening libraries (Oxamflatin, Scriptaid) were discovered to have a common structure with TSA and SAHA; i.e. a hydroxamic acid zinc- 
binding group linked via a spacer $\left(5\right.$ or $6 \mathrm{CH}_{2}$ ) to a hydrophobic group (14). Using an immunoblotting assay of histone deacetylation, Su et al demonstrated that Scriptaid is a potent HDACI with a >100-fold increase in histone acetylation, with relatively low toxicity (14).

This study was designed to define the biological and therapeutic effects of a novel HDACI, Scriptaid, which is recognized as the least toxic HDACI, in treating endometrial and ovarian cancers. We examined whether this compound was able to mediate the inhibition of cell growth, cell cycle arrest, apoptosis, and the expression of genes related to the malignant phenotype in endometrial and ovarian cancer cell lines.

\section{Materials and methods}

Cell lines. The Ishikawa human cell line was kindly provided by Dr Masato Nishida (Tsukuba University, Ibaraki, Japan), and the SK-OV-3 human cell line was obtained from the American Type Culture Collection (Manassas, VA). The Ishikawa cells were maintained as monolayers at $37^{\circ} \mathrm{C}$ in $5 \%$ $\mathrm{CO}_{2}$ /air in Dulbecco's modified Eagle's medium (DMEM) (Gibco, Rockville, MD) containing 5\% heat-inactivated fetal bovine serum (FBS) (Omega, Tarzana, CA). The SK-OV-3 cells were maintained as monolayers at $37^{\circ} \mathrm{C}$ in $5 \% \mathrm{CO}_{2}$ /air in Roswell Park Memorial Institute (RPMI)-1640 (Gibco) containing $10 \%$ heat-inactivated FBS (Omega).

Normal endometrial epithelial cells. Normal endometrial specimens were obtained from ten pre-menopausal patients who had undergone hysterectomies for leiomyoma. All patients had been free of any hormonal treatments before the operation. All of the specimens were diagnosed as being from the late proliferative phase (11-13th day of the menstrual cycle) using a standard histological examination of endometrial tissue. This study was approved by the institutional review board (IRB) of the Faculty of Medicine, Oita University, and written informed consent was obtained from all patients.

Normal endometrial epithelial cells were separated from stromal cells by digesting the tissue fragments with collagenase. Briefly, the tissue was minced into 2- to 3-mm pieces and incubated with collagenase (200 U/ml) (Gibco) in RPMI-1640 medium (Gibco) with stirring for $2 \mathrm{~h}$ at $37^{\circ} \mathrm{C}$. The suspension was then filtered through a $150-\mu \mathrm{m}$ wire sieve to remove mucus and undigested tissue. The filtrate was then passed through an $80-\mu \mathrm{m}$ wire sieve, which allowed the stromal cells to pass through while the intact glands were retained. After being washed three times with serum-free RPMI-1640, normal human endometrial epithelial cells were transferred to culture flasks (Corning, New York, NY) at a density of $10^{6}$ cells/ml in RPMI-1640 supplemented with $10 \%$ heatinactivated FBS (Omega), streptomycin (100 U/ml) (Gibco), and penicillin $(100 \mathrm{U} / \mathrm{ml})(\mathrm{Gibco})$. After $16 \mathrm{~h}$, the attached cells, which were $>98 \%$ pure as analyzed by immunocytochemical staining with antibodies to keratin (Dako, Copenhagen, Denmark), vimentin (V9; Dako), factor VIII (Dako), and leukocyte common antigen (2B11 + PD7/26; Dako), were used for the experiments. The cultures were incubated at $37^{\circ} \mathrm{C}$ in an atmosphere of $5 \% \mathrm{CO}_{2}$ in air at $100 \%$ humidity.
Chemicals. Scriptaid, the 6-(1,3-dioxo- $1 H, 3 H$-benzo[de] isoquinolin-2-yl)-hexanoic acid hydroxyamide, was obtained from Biomol (Plymouth Meeting, PA). Scriptaid was dissolved in anhydrous dimethyl sulfoxide (DMSO) to a $100-\mathrm{mM}$ stock solution. Subsequent dilutions were made in $1 \mathrm{mM}$ fatty acidfree bovine serum albumin (BSA).

MTT assays. 3-(4,5-dimethylthiazol-2-yl)-2,5-diphenyltetrazolium bromide (MTT; Sigma, St. Louis, MO) was placed in solution with phosphate-buffered saline (PBS) $(5 \mathrm{mg} / \mathrm{ml})$ and was used to measure cellular proliferation. Cells $\left(1 \times 10^{3}\right)$ were incubated in $100 \mu \mathrm{l}$ of culture medium for $48 \mathrm{~h}$ in 96 -well plates, and $10 \mu \mathrm{l}$ of MTT solution was added. After $4 \mathrm{~h}$ of incubation, $50 \mu \mathrm{l}$ of solubilization solution (20\% SDS) was added, and cells were then incubated at $37^{\circ} \mathrm{C}$ for $16 \mathrm{~h}$. In this assay, MTT is cleaved to an orange formazan dye by metabolically active cells. The dye was directly quantified using an enzyme-linked immunoabsorbent assay reader at $540 \mathrm{~nm}$. All experiments were performed independently at least three times in triplicate per experimental point.

Cell cycle analysis by flow cytometry. The cell cycle was analyzed by flow cytometry after 2 days of culturing either with or without Scriptaid, as described $(15,16)$. Ishikawa cells $\left(5 \times 10^{4}\right)$ were exposed to Scriptaid in 6-well, flat-bottomed plates for $48 \mathrm{~h}$. Total cells, both in the suspension and adherent, were collected, washed, and suspended in cold PBS. Cells were fixed in chilled $75 \%$ methanol and stained with propidium iodine (PI). Analysis was performed immediately after staining using the CellFit program (Becton-Dickinson, San Jose, CA), whereby the S phase was calculated using an RFit model. All experiments were performed independently at least three times in triplicate per experimental point.

Measurement of apoptosis (flow cytometry analysis with the annexin V/propidium iodide assay). An early step in the process of cell death (apoptosis and necrosis) is the redistribution of phosphatidylserine (PS) from the inner leaflet to the outer leaflet of the plasma membrane due to the loss of membrane asymmetry (17). The externalized PS can be readily visualized by incubating intact cells with a fluorescent derivative of the protein annexin V, a phospholipid-binding protein. Propidium iodine (PI), a fluorochrome, is used to label DNA. Unlike necrotic cells, apoptotic cells do not lose their cell membrane integrity and are thus impermeable for dyes such as PI. Therefore, the combination of annexin $\mathrm{V}$ and PI staining permits the simultaneous quantification of vital, apoptotic, and necrotic cells.

Cells were plated and grown overnight until they reached $80 \%$ confluence, and then treated with Scriptaid. After $48 \mathrm{~h}$, detached cells in the medium were collected, and the remaining adherent cells were harvested by trypsinization. The cells $\left(1 \times 10^{5}\right)$ were washed with PBS and resuspended in $250 \mu 1$ of binding buffer (annexin V-FITC kit; Becton-Dickinson) containing $10 \mu \mathrm{l}$ of $20 \mu \mathrm{g} / \mathrm{ml} \mathrm{PI}$ and $5 \mu \mathrm{l}$ of annexin $\mathrm{V}$ FITC, which binds to phosphatidylserine translocated to the exterior of the cell membrane early in the apoptosis pathway as well as during necrosis. After incubation for $10 \mathrm{~min}$ at room temperature in a light-protected area, the samples were analyzed on a FACScalibur flow cytometer (Becton- 
Dickinson). FITC and PI emissions were detected in the FL1 and FL2 channels, respectively. For each sample, data from 30,000 cells were recorded in list mode on logarithmic scales. Subsequent analysis was performed using CellQuest software (Becton-Dickinson). We could discriminate intact cells (annexin $\left./ \mathrm{PI}^{-}\right)$from apoptotic cells $\left(\right.$annexin $\left.^{+} / \mathrm{PI}^{-}\right)$and necrotic cells (annexin ${ }^{+} / \mathrm{PI}^{+}$) after treatment with Scriptaid. All experiments were performed independently at least three times in triplicate per experimental point.

Mitochondrial transmembrane potential. The electron gradient across the mitochondrial membrane space during normal respiration is called the mitochondrial transmembrane potential (MTP). Disruption of the MTP is one of the earliest intracellular events following the induction of apoptosis. The MitoCapture Apoptosis Detection Kit (Biovision Research Products, Palo Alto, CA) provides a simple, fluorescent-based method for distinguishing between healthy and apoptotic cells by detecting the changes in MTP. The kit utilizes MitoCapture, a cationic dye that fluoresces differently in healthy and apoptotic cells. In healthy cells, MitoCapture accumulates and aggregates in the mitochondria, giving off a bright red fluorescence. In apoptotic cells, MitoCapture cannot aggregate in the mitochondria due to the altered mitochondrial transmembrane potential, and it therefore remains in the cytoplasm in its monomer form, fluorescing green. The fluorescent signals can be easily detected by fluorescence microscopy using a band-pass filter (detects FITC and rhodamine) or analyzed by flow cytometry using the FITC channel for green monomers $(525 \mathrm{~nm})$ and the PI channel for red aggregates $(575 \mathrm{~nm})$.

Cells were prepared for FACS as described above and stained using the MitoCapture apoptosis detection kit obtained from Biovision with a fluorescent lipophilic cationic reagent that assesses mitochondrial membrane permeability, according to the manufacturer's recommendation. Briefly, cells were incubated with the MitoCapture reagent at $37^{\circ} \mathrm{C}$ in a $5 \% \mathrm{CO}_{2}$ incubator for $15 \mathrm{~min}$. This assay uses an intramitochondrial dye, which forms aggregates in healthy cells, leading to increased 575 fluorescence (PI channel, FL2) and indicating a normal MTP. In apoptotic cells, however, the dye is excluded from the mitochondria, leading to a loss of 575 fluorescence (aggregate) and an increase in 525 fluorescence (FITC channel, FL1) (monomer). Data were converted to density plots using System 2 software. In some experiments, FACS data were confirmed by fluorescent microscopy using a wide band pass filter. Cells with intact mitochondria exhibited focal red cytosolic fluorescence, whereas cells with permeabilized mitochondria exhibited diffuse green cytosolic fluorescence. Cells lacking red fluorescence and having green fluorescence were scored positive. All experiments were performed independently at least three times in triplicate per experimental point.

Western blot analysis. Cells were washed twice in PBS, suspended in lysis buffer [50 mM Tris ( $\mathrm{pH} \mathrm{8.0),} 150 \mathrm{mM}$ $\mathrm{NaCl}, 0.1 \%$ SDS, $0.5 \%$ sodium deoxycholate, $1 \%$ NP40, phenylmethylsulfonyl fluoride at $100 \mu \mathrm{g} / \mathrm{ml}$, aprotinin at $2 \mu \mathrm{g} /$ $\mathrm{ml}$, pepstatin at $1 \mu \mathrm{g} / \mathrm{ml}$, and leupeptin at $10 \mu \mathrm{g} / \mathrm{ml}$, and placed on ice for $30 \mathrm{~min}$. After centrifugation at $15,000 \mathrm{x} \mathrm{g}$ for $15 \mathrm{~min}$ at $4^{\circ} \mathrm{C}$, the suspension was collected. Protein

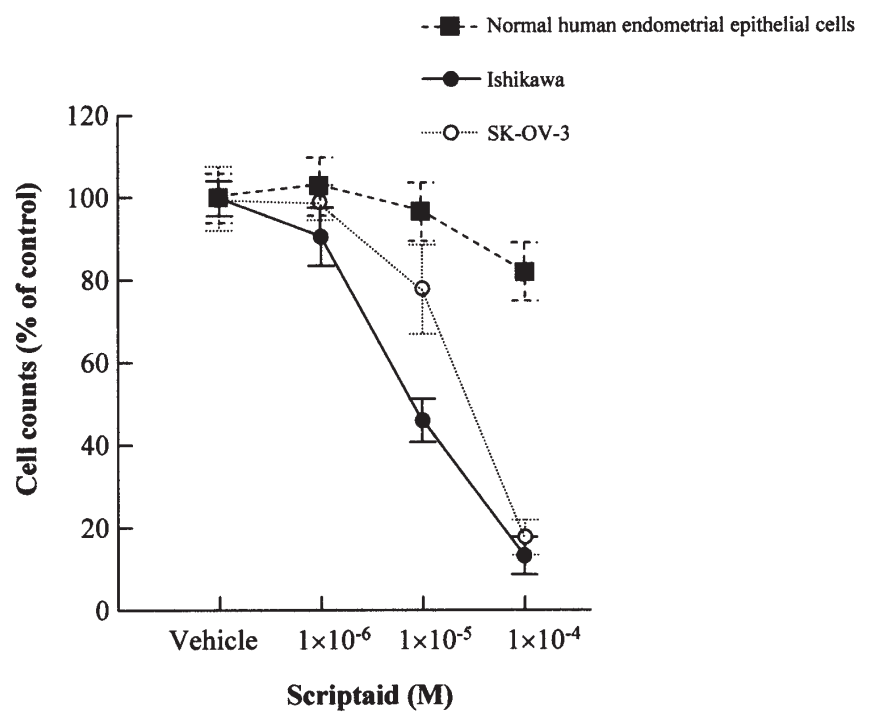

Figure 1. Effect of Scriptaid on the growth of endometrial cancer cell lines, ovarian cancer cell lines, and normal human endometrial epithelial cells in vitro. Ishikawa endometrial cancer cells, SK-OV-3 ovarian cancer cells, and normal human endometrial epithelial cells were treated with either Scriptaid at various concentrations $\left(1 \times 10^{-6}-1 \times 10^{-4} \mathrm{M}\right)$ or the dilutant (control) for $48 \mathrm{~h}$, and growth (\% of control) was measured by 3-(4,5-dimethylthiazol2-yl)-2,5-diphenyltetrazolium bromide assay. Results represent the mean $\pm \mathrm{SD}$ of three independent experiments with triplicate dishes.

concentrations were quantified using the Bio-Rad protein assay dye reagent concentrate (Bio-Rad Laboratories, Hercules, $\mathrm{CA}$ ) according to the manufacturer's recommendation. Whole cell lysates $(40 \mu \mathrm{g})$ were resolved by SDS-PAGE in a $4-15 \%$ gel, transferred to a polyvinylidene difuride membrane (Immobilon; Amersham Corp., Arlington Heights, IL), and probed sequentially with antibodies against acetylated H3 (1:1,000, Upstate, Lake Placid, NY), acetylated H4 (1:1,000, Upstate), p2 $1^{\text {WAF1 }}$ (Ab-1, 1:1,000, Oncogene, San Diego, CA), p27KIP1 (C-19, 1:1,000, Santa Cruz, CA), cyclin A (clone 25, 1:1,000, BD PharMingen, San Jose, CA), E-cadherin (G10, 1:1,000, Santa Cruz, CA), bcl-2 (100, 1:1,000, Santa Cruz, CA), and anti-GAPDH mAb (Research Diagnostics, Flanders, NJ). The blots were developed using an enhanced chemoluminescence (ECL) kit (Amersham).

Statistical analysis. All numerical data were expressed as the average of the values obtained \pm SD. Significance was determined by conducting a paired Student's t-test.

\section{Results}

Effects of Scriptaid on the proliferation and viability of the normal human endometrial epithelial cells, the Ishikawa endometrial cancer cell line, and the $S K-O V-3$ ovarian cancer cell line in vitro. We examined the antitumor effects of Scriptaid on normal human endometrial epithelial cells, the Ishikawa endometrial cancer cell line, and the SK-OV-3 ovarian cancer cell line in vitro, using an MTT assay with an exposure of 2 days to the Scriptaid (Fig. 1). Ishikawa endometrial cancer cells and SK-OV-3 ovarian cancer cells showed significant sensitivity to Scriptaid with $9.0 \times 10^{-6} \mathrm{M}$ and $5.5 \times 10^{-5} \mathrm{M}$, respectively, which caused a $50 \%$ inhibition 
A

Ishikawa cells

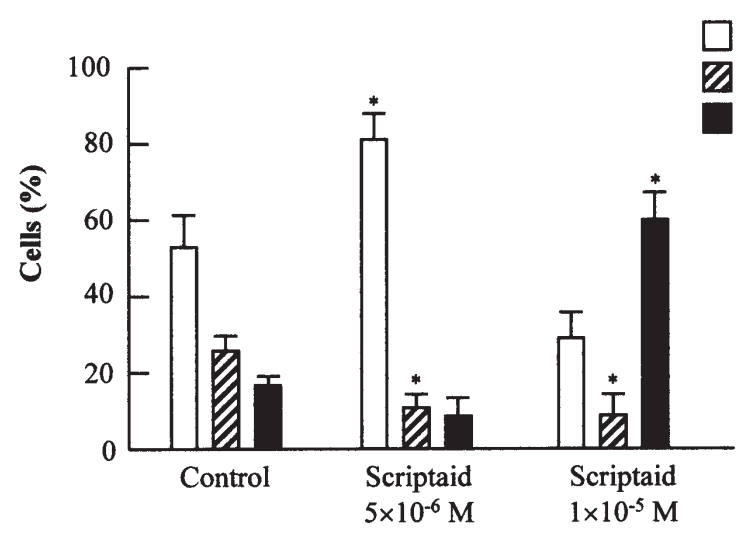

B

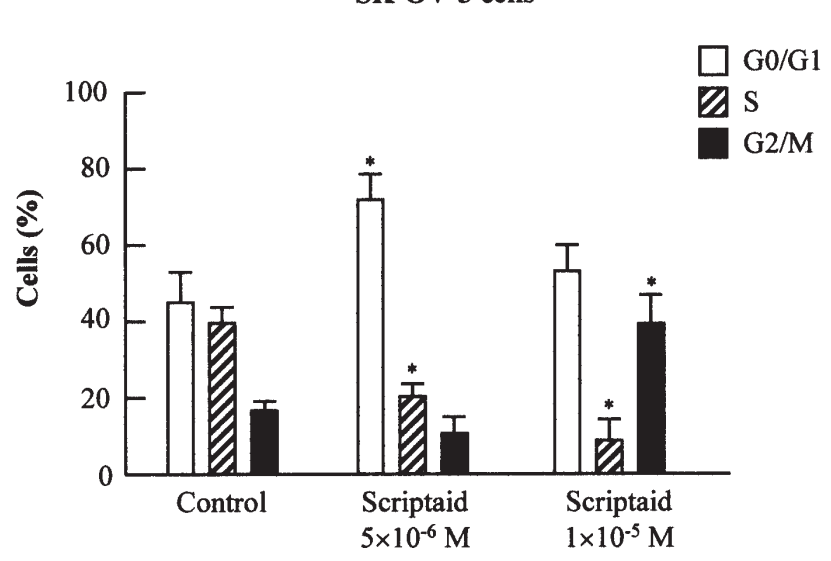

Figure 2. Cell-cycle analysis of Ishikawa cells and SK-OV-3 cells by flow cytometry. Ishikawa cells and SK-OV-3 cells were cultured with Scriptaid for 48 h, harvested, and stained with propidium iodine (PI). Control cells were treated with vehicle alone. Cell cycle analysis was performed by flow cytometry (see Materials and methods). Results represent the mean \pm SD of three independent experiments. ${ }^{*} \mathrm{p}<0.05$ as determined by the Student's t-test, difference of experimental compared with the control group.
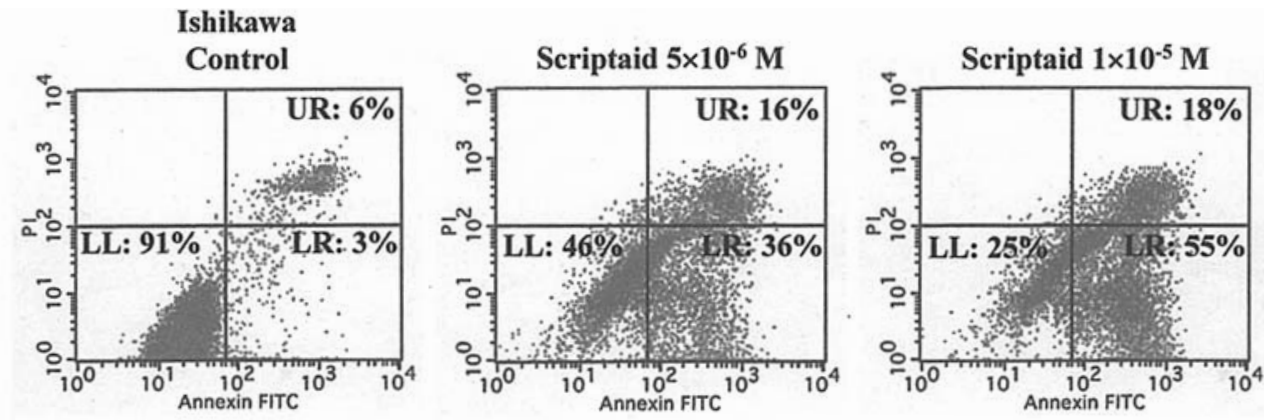

Figure 3. Cell death measured by annexin V and PI staining detected by flow cytometry. Ishikawa cells were treated with $5 \times 10^{-5} \mathrm{M}$ of Scriptaid for $48 \mathrm{~h}$. Cells were then stained with annexin V and PI. The positive cells were detected by flow cytometry. The viable cells were negative for both annexin V and PI staining (the lower left quadrant of the cytograms, LL), apoptotic cells were positive for annexin V staining while negative for PI staining (the lower right quadrant, LR), and necrotic cells were positive for both annexin V and PI staining (the upper right quadrant, UR). Each experiment was repeated three times. Two typical flow cytometry results are shown. The left figure shows histograms for untreated cells, and the middle figure and the right figure show those for treated cells at $48 \mathrm{~h}$.

$\left(\mathrm{ED}_{50}\right)$ of their growth. On the other hand, the normal human endometrial epithelial cells showed little sensitivity to Scriptaid from $1.0 \times 10^{-6} \mathrm{M}$ to $1 \times 10^{-4} \mathrm{M}$.

Cell-cycle analysis of endometrial cancer cells and ovarian cancer cells after exposure to Scriptaid. Endometrial cancer cells and ovarian cancer cells cultured for 2 days in the presence of Scriptaid showed an accumulation in the G0/G1 phase

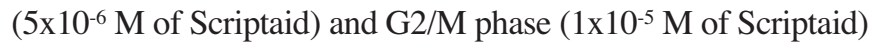
of the cell cycle, with a concomitant decrease in the proportion of those in the $\mathrm{S}$ phase (Fig. 2).

A total of $54.1 \pm 9.2 \%$ of the untreated Ishikawa cells, compared with $79.5 \pm 5.5 \%$ of cells cultured with $5 \times 10^{-6} \mathrm{M}$ of Scriptaid, were in the G0/G1 phase. A total of $18.9 \pm 1.0 \%$ of the untreated Ishikawa cells, compared with $60.6 \pm 6.8 \%$ of cells cultured with $1 \times 10^{-5} \mathrm{M}$ of Scriptaid, were in the G2/M phase. A total of $26.4 \pm 5.1 \%$ of the Ishikawa untreated cells, compared with $12.2 \pm 4.1 \%$ of cells cultured with $5 \times 10^{-6} \mathrm{M}$ of Scriptaid and $9.2 \pm 5.1 \%$ of those cultured with $1 \times 10^{-5} \mathrm{M}$ of Scriptaid, were in the $\mathrm{S}$ phase (Fig. 2A).

A total of $43.7 \pm 9.6 \%$ of the untreated SK-OV-3 cells, compared with $71.7 \pm 5.6 \%$ of cells cultured with $5 \times 10^{-6} \mathrm{M}$ of
Scriptaid, were in the G0/G1 phase. A total of $17.9 \pm 1.8 \%$ of the untreated SK-OV-3 cells, compared with $39.7 \pm 7.1 \%$ of cells cultured with $1 \times 10^{-5} \mathrm{M}$ of Scriptaid, were in the G2/M phase. A total of $39.9 \pm 3.2 \%$ of the SK-OV-3 untreated cells, compared with $21.0 \pm 2.7 \%$ of cells cultured with $5 \times 10^{-6} \mathrm{M}$ of Scriptaid and $9.2 \pm 6.1 \%$ of those cultured with $1 \times 10^{-5} \mathrm{M}$ of Scriptaid, were in the S phase (Fig. 2B).

Apoptotic changes in Scriptaid-treated endometrial cancer cells and ovarian cancer cells. To assess the capability of endometrial cancer cells and ovarian cancer cells to undergo apoptosis in response to drug exposure and to help distinguish between different types of cell death, we double-stained Scriptaid-treated cells with annexin V and PI and analyzed the results using flow cytometry. Annexin V binding combined with PI labeling was performed for the distinction of early apoptotic (annexin $\mathrm{V}^{+}$/propidium iodide) and necrotic (annexin $\mathrm{V}^{+} /$propidium iodide ${ }^{+}$cells. At increasing doses of Scriptaid, we also detected a simultaneous increase in both the annexin $\mathrm{V}^{+} /$propidium iodide- fraction (early apoptotic) and annexin $\mathrm{V}^{+} /$propidium iodide ${ }^{+}$(regarded as necrotic) subpopulations (Fig. 3). After incubations with death stimuli $\left(1 \times 10^{-5} \mathrm{M}\right.$ of 
Table I. Cell death measured by annexin V and PI staining detected by flow cytometry in Ishikawa endometrial cancer cells.

\begin{tabular}{lrrr}
\hline & Vehicle & $\begin{array}{r}\text { Scriptaid } \\
5 \times 10^{-6} \mathrm{M}\end{array}$ & $\begin{array}{r}\text { Scriptaid } \\
1 \times 10^{-5} \mathrm{M}\end{array}$ \\
\hline Viable (LL) (\%) & $90.5 \pm 2.2$ & $46.4 \pm 3.0$ & $23.8 \pm 3.5$ \\
Apoptosis (LR) (\%) & $3.0 \pm 0.9$ & $38.1 \pm 5.2$ & $56.1 \pm 6.8$ \\
Necrosis (UR) (\%) & $5.8 \pm 1.8$ & $16.3 \pm 3.0$ & $19.9 \pm 3.7$
\end{tabular}

The viable cells were negative for both annexin V and PI staining (the lower left quadrant of the cytograms, LL), apoptotic cells were positive for annexin V staining while negative for PI staining (the lower right quadrant, LR), and necrotic cells were positive for both annexin V and PI staining (the upper right quadrant, UR). Each experiment was repeated three times. Data show the mean \pm SD.

Table III. Apoptotic cells measured by mitochondrial transmembrane potential in Ishikawa endometrial cancer cells.

\begin{tabular}{lrrr}
\hline & Vehicle & $\begin{array}{r}\text { Scriptaid } \\
5 \times 10^{-6} \mathrm{M}\end{array}$ & $\begin{array}{r}\text { Scriptaid } \\
1 \times 10^{-5} \mathrm{M}\end{array}$ \\
\hline Viable $(\%)$ & $97.5 \pm 0.8$ & $78.8 \pm 1.1$ & $47.8 \pm 3.2$ \\
Apoptosis $(\%)$ & $2.5 \pm 0.5$ & $21.2 \pm 0.9$ & $52.0 \pm 2.8$ \\
\hline
\end{tabular}

Table II. Cell death measured by annexin V and PI staining detected by flow cytometry in SK-OV-3 ovarian cancer cells.

\begin{tabular}{lrrr}
\hline & Vehicle & $\begin{array}{r}\text { Scriptaid } \\
5 \times 10^{-6} \mathrm{M}\end{array}$ & $\begin{array}{r}\text { Scriptaid } \\
1 \times 10^{-5} \mathrm{M}\end{array}$ \\
\hline Viable (LL) (\%) & $92.1 \pm 3.0$ & $68.1 \pm 5.7$ & $29.3 \pm 4.6$ \\
Apoptosis (LR) (\%) & $2.5 \pm 0.7$ & $20.8 \pm 3.1$ & $53.0 \pm 5.7$ \\
Necrosis (UR) (\%) & $5.1 \pm 1.1$ & $11.5 \pm 2.5$ & $17.8 \pm 3.8$ \\
\hline
\end{tabular}

The viable cells were negative for both annexin V and PI staining (the lower left quadrant of the cytograms, LL), apoptotic cells were positive for annexin V staining while negative for PI staining (the lower right quadrant, LR), and necrotic cells were positive for both annexin V and PI staining (the upper right quadrant, UR). Each experiment was repeated three times. Data show the mean \pm SD.

Table IV. Apoptotic cells measured by mitochondrial transmembrane potential in SK-OV-3 ovarian cancer cells.

\begin{tabular}{lrrr}
\hline & Vehicle & $\begin{array}{l}\text { Scriptaid } \\
5 \times 10^{-6} \mathrm{M}\end{array}$ & $\begin{array}{l}\text { Scriptaid } \\
1 \times 10^{-5} \mathrm{M}\end{array}$ \\
\hline Viable $(\%)$ & $96.9 \pm 0.5$ & $84.9 \pm 2.6$ & $48.5 \pm 5.6$ \\
Apoptosis $(\%)$ & $3.0 \pm 0.2$ & $14.8 \pm 1.2$ & $51.6 \pm 4.8$ \\
\hline
\end{tabular}

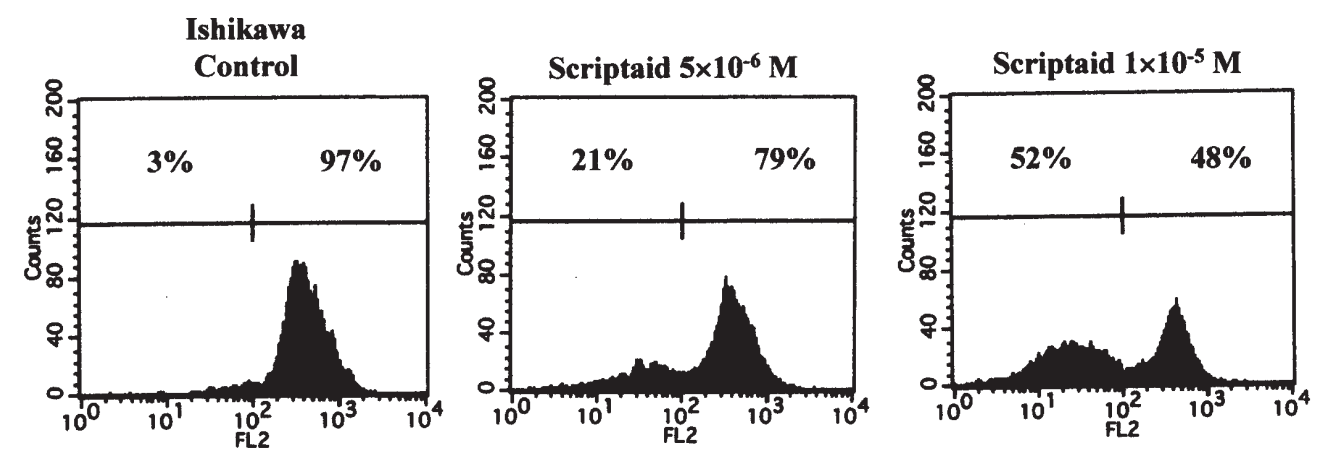

Figure 4. The effect of Scriptaid treatment on mitochondrial transmembrane potential. Mitochondrial transmembrane potential (MTP) was analyzed using the MitoCapture assay, as described in Materials and methods. Ishikawa cells were treated with Scriptaid for $48 \mathrm{~h}$ and harvested for flow cytometry. Each histogram profiles the number of cells relative to their fluorescent intensity. In healthy cells, intramitochondrial dye forms aggregates, leading to increased FL2 fluorescence. In apoptotic cells, the dye is excluded from the mitochondria, leading to a loss of FL2 fluorescence.

Scriptaid) for 48 h, $56.1 \pm 6.8 \%$ of Ishikawa cells were annexin $\mathrm{V}^{+} /$propidium iodide - However, a similar pattern of labeling was detectable in approximately $3.0 \%$ of Ishikawa cells incubated under normal conditions. The percentage of annexin $\mathrm{V}^{+}$/propidium iodide ${ }^{+}$Ishikawa cells was $19.9 \pm 3.7 \%$ in cultures exposed to noxious stimuli ( $1 \times 10^{-5} \mathrm{M}$ of Scriptaid) for $48 \mathrm{~h}$, but $<6 \%$ in Ishikawa cells incubated under the normal condition. A typical cyto-diagram obtained by flow cytometry and CellQuest software is shown in Fig. 3, and the results (mean \pm SD in triplicate) are shown in Tables I and II.

Loss of mitochondrial membrane potential in response to Scriptaid. Loss of MTP has been shown to occur prior to nuclear condensation and caspase activation and is linked to cytochrome $c$ release in many, but not all, apoptotic cells $(18,19)$. Using MitoCapture staining and flow cytometry, we analyzed the MTP in Scriptaid-treated Ishikawa cells and SK-OV-3 cells. Intracellular fluorescence was assayed by FACS after loading cells with an intramitochondrial dye. High fluorescence at $575 \mathrm{~nm}$ (FL2) corresponds to the aggregated form of the dye and is proportional to an intact MTP, whereas loss of MTP leads to a loss of 575 fluorescence and an increase in fluorescence at $525 \mathrm{~nm}$ (FL1) (monomeric form of the dye). As seen in Fig. 4, untreated cells exhibit high 575 fluorescence, indicating normal MTP. Treatment of cells with Scriptaid results in a loss of 575 fluorescence and an increase in fluorescence at 525, indicating a loss of MTP (Fig. 4, Tables III and IV). 
A



B

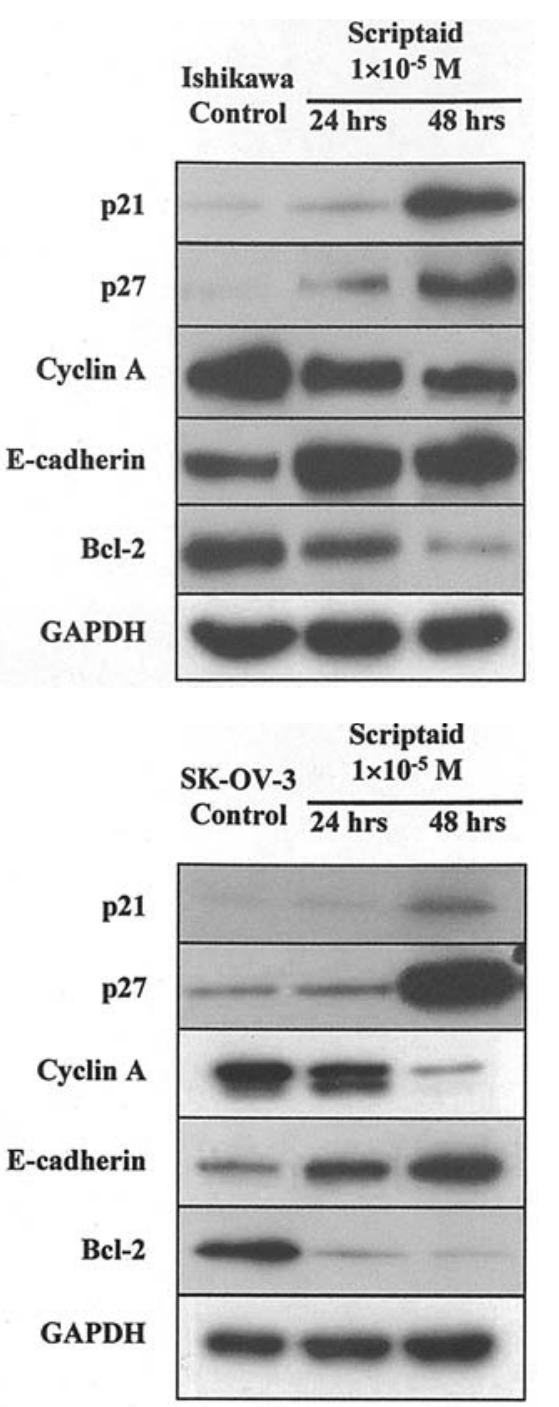

Figure 5. Cell cycle- and apoptosis-related protein expression in Ishikawa cells and SK-OV-3 cells, as measured by Western blot analysis. Ishikawa cells and SK-OV-3 cells were treated with $1 \times 10^{-5} \mathrm{M}$ Scriptaid, and cell lysates were harvested after 24 and $48 \mathrm{~h}$. Western blot analysis was performed with a series of antibodies. (A) Acetylated histone H3, acetylated histone H4; (B and C) p $21^{\mathrm{WAF} 1}, \mathrm{p} 27^{\mathrm{KIP} 1}$, cyclin A, E-cadherin, and bcl-2. Control cells were treated with vehicle alone. The amount of protein was normalized by comparison to levels of GAPDH.

Effect of Scriptaid on the acetylation of histones. Treatment of Ishikawa endometrial cancer cells and SK-OV-3 ovarian cancer cells with Scriptaid dramatically increased the levels of acetylated H3 and H4 (Ishikawa, Fig. 5A; SK-OV-3, data not shown).
Effect of Scriptaid on the expression of cell-cycle and apoptosis-related proteins as well as E-cadherin. p21 $1^{\mathrm{WAF} 1}$ and $\mathrm{p} 27^{\mathrm{KIP} 1}$ are cyclin-dependent kinase inhibitors (CDKIs) that bind to cyclin-dependent kinase complexes and decrease kinase activity, and they may act as key regulators of the G0/G1 accumulation (reviewed in refs. 15,16). We examined the effect of Scriptaid on the expression of $\mathrm{p} 21^{\mathrm{WAF} 1}$ and p27 ${ }^{\mathrm{KIP} 1}$ in Ishikawa cells and SK-OV-3 cells by Western blot analysis (Fig. 5B and C). Scriptaid markedly upregulated the level of $\mathrm{p} 21^{\mathrm{WAF} 1}$ and $\mathrm{p} 27^{\mathrm{KIP} 1}$ proteins, which were expressed at negligible levels in the untreated Ishikawa endometrial cancer cell line and SK-OV-3 ovarian cancer cell line. Conversely, Scriptaid $\left(1 \times 10^{-5} \mathrm{M}\right)$ decreased the levels of cyclin A by $50 \%$ and decreased bcl-2 levels by $20 \%$ in the Ishikawa cells (Fig. 5B). It also lowered the expression of cyclin A by $25 \%$ and caused a $10 \%$ decrease in bcl-2 expression in the SKOV-3 cells (Fig. 5C).

E-cadherin binds to $B$-catenin and can act as a tumor suppressor gene; its promoter has $\mathrm{CpG}$ islands that are frequently methylated in selected cancers. Scriptaid markedly increased the expression level of E-cadherin in both Ishikawa and SK-OV-3 cells (Fig. 5B and C).

\section{Discussion}

Using a high-throughput system based on a stably integrated transcriptional reporter to screen a library of 16,320 compounds (DIVERset, Chembridge, San Diego, CA), Su et al identified a novel HDAC inhibitor, Scriptaid (14). Nullscript, which possesses a shorter side-chain (3C) than Scriptaid (5C) between the tricyclic core and the carbonyl group, was inactive in transcriptional facilitation. This confirms that there is a minimal requirement for the length of the linker chain for HDAC inhibition. Scriptaid conferred the greatest effect on augmentation of the signal transduction TGFß pathway, including a number of human suppressor genes such as SMAD4 (14).

Epigenetic mechanisms, such as DNA methylation and histone deacetylation, may play a role in the proliferation of human cancer cells. HDACIs can prevent proliferation and induce differentiation of numerous transformed cell types, including neuroblastomas, erythroleukemia, acute myelogenous leukemia, and carcinomas of the skin, breast, prostate, bladder, lung, colon, cervix, endometrium, and ovary (20). The effect of Scriptaid in human cancers, however, has not been fully examined. A recent study by Keen et al indicated that Scriptaid had a significant growth-suppressing effect on ER-negative human breast cancer cells (21). This stimulated us to examine the effect of Scriptaid on endometrial and ovarian cancer cell lines (Ishikawa and SK-OV-3).

We have demonstrated that Scriptaid is highly effective in suppressing the growth of human endometrial and ovarian cancer cells, although Scriptaid has little effect on normal human endometrial epithelial cells. These events are associated with the accumulation of acetylated $\mathrm{H} 3$ and $\mathrm{H} 4$ histone proteins, confirming that Scriptaid acts as an HDAC inhibitor in these human cancer cell lines. The prominent arrest of cancer cells in the G0/G1 phase of the cell cycle is likely to account for this effect. p21 $1^{\mathrm{WAF} 1}$ and p27 $7^{\mathrm{KIP} 1}$ are cyclin-dependent kinase inhibitors that have important roles in blocking the 
cell cycle in the G1 phase (22). The protein levels of both $\mathrm{p} 21^{\mathrm{WAF} 1}$ and $\mathrm{p} 27^{\mathrm{KIP} 1}$ increased following the treatment of endometrial and ovarian cancer cells with Scriptaid, supporting their contribution as a possible mechanism by which these agents inhibit endometrial and ovarian cancer growth.

Our results show that Scriptaid decreases the levels of cyclin A, apparently at the transcription level. Therefore, Scriptaid causes decreased expression of cyclin A and increased expression of $\mathrm{p} 21^{\mathrm{WAF} 1}$, which probably combine to modulate the activity of the downstream $\mathrm{pRb} / \mathrm{E} 2 \mathrm{~F}$ axis, triggering cell cycle arrest (23).

We show that treatment with Scriptaid dramatically and significantly increased the number of apoptotic cells in endometrial and ovarian cancer cell lines. This effect was associated with a decrease in levels of the anti-apoptotic protein, bcl-2.

The E-cadherin gene is involved in cell-cell adhesion, and loss of function has been associated with enhanced metastatic growth of tumor cells (24). Inactivation of this gene by hypermethylation has been observed in breast carcinoma cells and in primary breast tumors (25). We find that transcription of E-cadherin is upregulated in endometrial and ovarian cancer cells treated with Scriptaid, suggesting a gain of tumor suppressor function in response to inhibition of histone deacetylase.

In summary, Scriptaid exhibits antiproliferative activity and potently induces apoptosis in human endometrial and ovarian cancer cells. These events are accompanied by induction of $\mathrm{p} 21^{\mathrm{WAF} 1}$ and $\mathrm{p} 27^{\mathrm{KIP} 1}$ and down-regulation of several anti-apoptosis and cell cycle-related proteins; bcl-2, cyclin A, and E-cadherin. The present findings raise the possibility that Scriptaid may prove particularly effective in the treatment of endometrial and ovarian cancers.

\section{Acknowledgments}

The study was supported by a Grant-in-Aid (no. 16790961 to N.T.) for Scientific Research from the Ministry of Education, Culture, Sports, Science, and Technology, Japan.

\section{References}

1. Strahl BD and Allis CD: The language of covalent histone modifications. Nature 403: 41-45, 2000.

2. Verdin E, Dequiedt F and Kasler HG: Class II histone deacetylases: versatile regulators. Trends Genet 19: 286-293, 2003.

3. Laherty CD, Yang WM, Sun JM, Davie JR, Seto E and Eisenman RN: Histone deacetylases associated with the mSin3 corepressor mediate mad transcriptional repression. Cell 89: 349-356, 1997.

4. Dhordain P, Lin RJ, Quief S, Lantoine D, Kerckaert JP, Evans RM and Albagli O: The LAZ3(BCL-6) oncoprotein recruits a SMRT/mSIN3A/histone deacetylase containing complex to mediate transcriptional repression. Nucleic Acids Res 26: 4645-4651, 1998.

5. Gelmetti V, Zhang J, Fanelli M, Minucci S, Pelicci PG and Lazar MA: Aberrant recruitment of the nuclear receptor corepressor-histone deacetylase complex by the acute myeloid leukemia fusion partner ETO. Mol Cell Biol 18: 7185-7191, 1998.
6. Yoshida M, Kijima M, Akita M and Beppu T: Potent and specific inhibition of mammalian histone deacetylase both in vivo and in vitro by trichostatin A. J Biol Chem 265: 17174-17179, 1990.

7. Yoshida M, Hoshikawa Y, Koseki K, Mori K and Beppu T: Structural specificity for biological activity of trichostatin A, a specific inhibitor of mammalian cell cycle with potent differentiation-inducing activity in Friend leukemia cells. J Antibiot 43: 1101-1106, 1990.

8. Yoshida M, Horinouchi S and Beppu T: Trichostatin A and trapoxin: novel chemical probes for the role of histone acetylation in chromatin structure and function. Bioessays 17: 423-430, 1995.

9. Marks PA, Richon VM and Rifkind RA: Histone deacetylase inhibitors: inducers of differentiation or apoptosis of transformed cells. J Natl Cancer Inst 92: 1210-1216, 2000.

10. Zhou Q, Melkoumian ZK, Lucktong A, Moniwa M, Davie JR and Strobl JS: Rapid induction of histone hyperacetylation and cellular differentiation in human breast tumor cell lines following degradation of histone deacetylase-1. J Biol Chem 27: 35256-35263, 2000.

11. De Ruijter AJ, van Gennip AH, Caron HN, Kemp S and van Kuilenburg AB: Histone deacetylases (HDACs): characterization of the classical HDAC family. Biochem J 370: 737-749, 2003.

12. Warrell RP Jr, He LZ, Richon VM, Calleja E and Pandolfi PP: Therapeutic targeting of transcription in acute promyelocytic leukemia by use of an inhibitor of histone deacetylase. J Natl Cancer Inst 90: 1621-1625, 1998.

13. Newmark HL and Young CW: Butyrate and phenylacetate as differentiating agents: practical problems and opportunities. J Cell Biochem Suppl 22: 247-253, 1995.

14. Su GH, Sohn TA, Ryu B and Kern SE: A novel histone deacetylase inhibitor identified by high-throughput transcriptional screening of a compound library. Cancer Res 60: 3137-3142, 2000

15. Takai N, Desmond JC, Kumagai T, Gui D, Said JW, Whittaker S, Miyakawa I and Koeffler HP: Histone deacetylase inhibitors have a profound anti-growth activity in endometrial cancer cells. Clin Cancer Res 10: 1141-1149, 2004.

16. Takai N, Kawamata N, Gui D, Said JW, Miyakawa I and Koeffler HP: Human ovarian carcinoma cells: histone deacetylase inhibitors exhibit antiproliferative activity and potently induce apoptosis. Cancer 101: 2760-2770, 2004.

17. Rimon G, Bazenet CE, Philpott KL and Rubin LL: Increased surface phosphatidylserine is an early marker of neuronal apoptosis. J Neurosci Res 48: 563-570, 1997.

18. Chen Y, Kramer DL, Diegelman P, Vujcic S and Porter CW: Apoptotic signaling in polyamine analogue-treated SK-MEL-28 human melanoma cells. Cancer Res 61: 6437-6444, 2001.

19. Sandberg EM and Sayeski PP: Jak2 tyrosine kinase mediates oxidative stress-induced apoptosis in vascular smooth muscle cells. J Biol Chem 279: 34547-34552, 2004.

20. Richon VM, Sandhoff TW, Rifkind RA and Marks PA: Histone deacetylase inhibitor selectively induces p21WAF1 expression and gene-associated histone acetylation. Proc Natl Acad Sci USA 97: 10014-10019, 2000.

21. Keen JC, Yan L, Mack KM, Pettit C, Smith D, Sharma D and Davidson NE: A novel histone deacetylase inhibitor, Scriptaid, enhances expression of functional estrogen receptor $\alpha$ (ER) in ER negative human breast cancer cells in combination with 5-aza 2'-deoxycytidine. Breast Cancer Res Treat 81: 177-186, 2003.

22. Johnson DG and Walker CL: Cyclins and cell cycle checkpoints. Annu Rev Pharmacol Toxicol 39: 295-312, 1999.

23. Freytag SO: Enforced expression of the c-myc oncogene inhibits cell differentiation by precluding entry into a distinct predifferentiation state in $\mathrm{G}_{0} / \mathrm{G}_{1}$. Mol Cell Biol 8: 1614-1624, 1988.

24. Takeichi M: Cadherin cells adhesion receptors as a morphogenetic regulator. Science 251: 1451-1455, 1991.

25. Graff JR, Herman JG, Lapidus RL, Chopra H, Xu R, Jarrard DF, Isaacs WB, Pitha PM, Davidson NE and Baylin SB: E-cadherin expression is silenced by DNA hypermethylation in human breast and prostate carcinomas. Cancer Res 55: 5195-5199, 1995 . 